\title{
Urbanización de la pobreza en el aglomerado Gran Salta (Noroeste Argentino).
} Sus manifestaciones actuales

Alejandra Carolina del Castillo

Doctora en Ciencias Sociales (orientación en Geografía), Universidad Nacional de Tucumán. Becaria posdoctoral del CONICET. Integrante del Instituto de Estudios Geográficos Dr. Guillermo Rohmeder.

delcale@hotmail.com

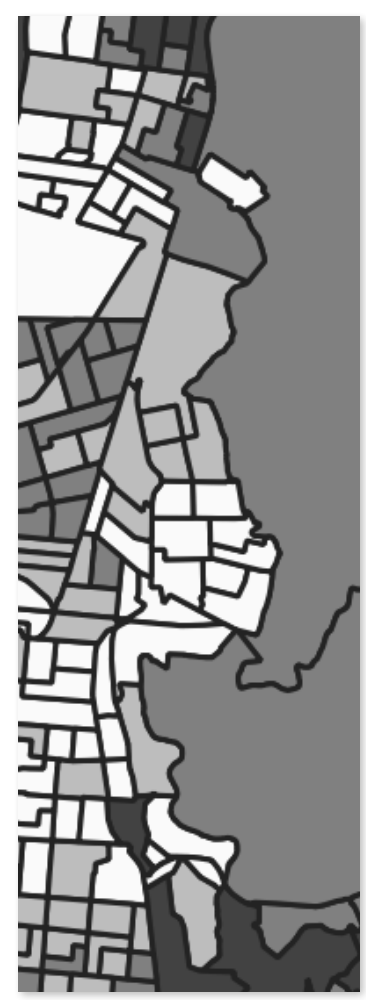

$10.30972 / \mathrm{crn} .28284323$ ISNN 1666-6186. Volumen 28 N. ${ }^{\circ} 28$ (junio de 2020) Pp. 035-058 - Recibido: 06-06-18. Evaluado y aprobado: 18-03-20 
Urbanización de la pobreza en el aglomerado Gran Salta (Noroeste Argentino). Sus manifestaciones actuales

\section{Resumen}

El presente trabajo se propone analizar la urbanización de la pobreza en el aglomerado Gran Salta y sus manifestaciones actuales desde una perspectiva histórica. Los interrogantes que orientan su desarrollo son: ¿cuáles son los principales procesos que incidieron en la urbanización de la pobreza en el aglomerado Gran Salta?; ¿cómo se manifestó espacialmente dicho proceso?; ¿qué magnitud adquiere la pobreza en los inicios del siglo XXI?; ¿cuáles son las características sociodemográficas de la población que reside en las áreas de mayor pobreza?

Los resultados evidencian que la urbanización de la pobreza tiene más de un siglo de desarrollo, pero desde la década de 1960 adquiere un desarrollo disruptivo. En la periferia de la ciudad se multiplican villas de emergencia y barrios de loteos económicos precarios. En los inicios del siglo XXI se observa un crecimiento sostenido de la pobreza, con el crecimiento de numerosos asentamientos informales, y un proceso de acumulación de desventajas para las poblaciones afectadas.

\section{Palabras clave}

Pobreza urbana; expansión urbana; Gran Salta.

\section{Urbanization of poverty in the Gran Salta agglomerate (Northwestern Argentina). Its current manifestations}

\begin{abstract}
This paper aims to analyze the urbanization of poverty in the Gran Salta agglomerate and its current manifestations from a historical perspective. The related research questions are as follows: What are the main processes that influenced the urbanization of poverty in the Gran Salta agglomerate? How was this process manifested spatially? What is the magnitude of poverty at the beginning of the 21st century? What are the sociodemographic characteristics of the population living in the highest poverty areas?

The results show that the urbanization of poverty has more than a century of development, but since the 1960s it has acquired a disruptive development. In the city's periphery, emergency slums settlement and precarious boroughs are multiplying. At the beginning of the 21st century a sustained increase in poverty, with a growth of many informal settlements and a process of accumulation of disadvantages for the affected populations is observed.
\end{abstract}

\section{Keywords}

Urban poverty; urban sprawl; Gran Salta.

\section{Urbanização da pobreza no aglomerado Gran Salta (Noroeste Agentino). Manifestações atuais}

\section{Resumo}

O presente trabalho se propõe analisar a urbanização da pobreza no aglomerado de Gran Salta e suas manifestações atuais sob uma perspectiva histórica. As questões que norteiam seu desenvolvimento são: Quais são os principais processos que influenciaram a urbanização da pobreza no aglomerado de Gran Salta?; como esse processo se manifestou espacialmente?; qual é a magnitude da pobreza no início do século XXI?; quais são as características sociodemográficas da população que reside nas áreas de maior pobreza? Os resultados mostram que a urbanização da pobreza tem mais de um século de desenvolvimento, mas desde a década de 1960 ela adquiriu um desenvolvimento disruptivo. Nos arredores da cidade, cidades emergentes e bairros de parcelas econômicas precárias se multiplicam. No início do século XXI, há um crescimento sustentado da pobreza, com o crescimento de numerosos assentamentos informais e um processo de acumulação de desvantagens para as populações afetadas.

\section{Palavras chave}

Pobreza urbana; expansão urbana; Gran Salta. 


\section{Introducción}

Gran Salta es uno de los aglomerados con mayores niveles de pobreza. Los datos de la Encuesta Permanente de Hogares (EPH), para el primer semestre de 2019, lo sitúan en el cuarto lugar, con el $41,8 \%$ de personas en condiciones de pobreza. Otro estudio basado en una metodología de medición multidimensional en 2015 lo ubicaba en el primer lugar, con un $40 \%$ de población con privaciones (ArÉvalo y PAZ, 2015) ${ }^{1}$.

Este proceso de deterioro social, lejos de remitirse a las últimas décadas, tiene un carácter estructural y se enmarca en lo que denominamos urbanización de la pobreza. Se inició a principios del siglo XX con una moderada pero continua migración rural, y adquirió un desarrollo disruptivo a partir de la década de 1960.

La ciudad de Salta, al igual que el resto de las capitales provinciales del Noroeste Argentino (NOA), experimentó un acelerado crecimiento de su población entre 1960 y mediados de 1990 (Ortiz de D’Arterio y Paolasso, 2005; Bolsi y Mignone, 2009). Se constituyó en receptora de migrantes internos, provenientes de áreas rurales o de pequeñas ciudades, como consecuencia de la crisis de las actividades agrarias (VAPÑARSKY, 1994; Di FABIo Rocca ET Ál., 2016). Estos se asentaron en la periferia urbana en las llamadas villas de emergencia o en loteos económicos precarios.

Desde la década de 1990 la pobreza urbana se expandió e intensificó en el aglomerado salteño como resultado de las reformas estructurales que agravaron, entre otras cuestiones, los problemas de empleo y habitacionales. Crecieron los asentamientos informales en diferentes zonas de la ciudad, principalmente en el sureste. La contracara de este proceso, durante este período, es el desarrollo de áreas de opulencia que acentuaron los contrastes sociales y agregaron desventajas a las poblaciones.

Teniendo en cuenta el carácter histórico de la urbanización de la pobreza, los interrogantes que guían el presente artículo son: ¿cuáles son los principales procesos que incidieron en la configuración de la pobreza en el aglomerado Gran Salta?; ¿cómo se manifestó espacialmente dicho proceso?; ¿qué magnitud adquiere la pobreza en los inicios del siglo XXI?; ¿cuáles son las características sociodemográficas de la población que reside en las áreas de mayor pobreza?
1. Utilizan un indicador que mide ingresos, vivienda, inclusión y salud. Para más información consultar en ARÉVALO Y PAZ (2015). 
2. El empleo informal se refiere a los trabajadores que no se encuentran registrados y que componen el denominado empleo "en negro". La precariedad laboral comprende a los trabajadores que no están sujetos a la legislación del trabajo, independientemente de si se desempeñan en el sector formal o informal de la economía (OIT, 2003).

\section{Consideraciones teóricas y metodológicas}

Partimos de considerar la pobreza como una situación estructural, y no como la suma de dimensiones o aspectos parciales de privación en la que se encuentran determinadas familias, producto de su particular inserción en la estructura socioproductiva, siendo esta la que determina la posibilidad de acceder en mayor o menor medida a determinados bienes y servicios (Moreno, 2009). Dentro de la complejidad del fenómeno de la pobreza, y considerando la dimensión territorial, nos centramos en la pobreza urbana en tanto adquiere manifestaciones distintivas respecto del medio rural.

Una característica del desarrollo de la pobreza que hay que tener en cuenta es que el número de pobres que viven en las ciudades tiende a ser cada vez mayor frente al promedio total de pobres, denominándose este proceso “urbanización de la pobreza” (ZICCARDI, 2008). El centro de gravedad de la pobreza se desplazó, durante la segunda mitad del siglo XX, del campo a la ciudad, con las crisis de las actividades agrarias de las economías regionales y las migraciones internas (АцтіміR, 1978).

Gran parte de estos contingentes de población que se desplazó a las ciudades encontró dificultades para insertarse plenamente en el sistema productivo. Los problemas de empleo - precariedad, bajos niveles salariales e informalidad - constituyen, en este sentido, una dimensión central en el análisis de la pobreza urbana (Ziccardi, 2008)². Otro componente es la falta de acceso al suelo urbano, a una vivienda digna y a los servicios e infraestructura urbanos (ARRiagada, 2000; Mac Donald, 2004; Ziccardi, 2008; Winchester, 2008). La respuesta al problema fue - y continúa siendo — el asentamiento informal en las zonas más degradadas de las ciudades, generalmente la periferia, en condiciones de inseguridad legal respecto de la tenencia de la tierra y hacinamiento habitacional (Cravino, 2008), entre otros aspectos que denotan vulnerabilidad.

A los aspectos arriba mencionados, con las transformaciones territoriales en las ciudades, se suman las desventajas asociadas a la fragmentación urbana y la intensificación de los procesos de segregación socioespacial. A la discontinuidad física y morfológica de las áreas de pobreza, con la consiguiente segmentación en la calidad de los servicios, se suman barreras simbólicas que suelen traducirse en una patologización de los espacios (barrios, escuelas, calles, etc.) (JANOschKA, 2002; BAYón, 2012). 
La descripción del proceso de urbanización de la pobreza en el aglomerado Gran Salta se realiza a partir de la revisión bibliográfica de estudios que abordan procesos y fenómenos como las configuraciones sociales, relaciones de tutela y los dispositivos de intervención en torno a la pobreza en la primera mitad del siglo XX; los cambios de las configuraciones sociales y la construcción de la identidad salteña a lo largo del siglo XX; la forma en que se produce la construcción social del hábitat popular; las transformaciones de carácter socioespacial producidas en Vaqueros - localidad que integra el aglomerado—; miradas y representaciones sobre la pobreza desde el estudio de la pobreza en la provincia de Salta en la década de 1990; la evolución de los aspectos urbanos y arquitectónicos de la ciudad de Salta (SBRocco, 1997; AgUILAR ET AL., 2002; Álvarez Leguizamón, 2004; Aguilar y Costilla, 2009; Álvarez Leguizamón et al., 2010; Gómez, 2017).

Para abordar las manifestaciones actuales de la pobreza en el aglomerado Gran Salta, se utiliza el Índice de Condiciones de Vida (ICVI) propuesto por PaOLASso et AL. (2019). Este, tomando como fuente de información los Censos Nacionales de Población, Hogares y Viviendas (CNHPyV) 2001 y 2010, mide variables vinculadas con la educación (población mayor a doce años que no sabe leer ni escribir; población mayor a cinco años que nunca asistió a un establecimiento educativo) y a la vivienda (inodoro sin descarga de agua; hacinamiento crítico; propiedad de vivienda y terreno precaria y uso de leña y carbón como el principal combustible usado para cocinar).

Debido a la imposibilidad de comparar los valores del ICVI a nivel de los radios censales entre 2001 y 2010, en tanto la cantidad y diseño son diferentes, se analizan por cuartiles. Se describen, luego, ciertas características sociodemográficas de la población que reside en los radios localizados en el cuartil IV, a partir de la información CNHPyV 2010, en tanto serían las áreas de mayor pobreza. Se comparan los valores hallados con los guarismos del Gran Salta, el cual oficia como un marco de referencia, para analizar las brechas existentes y los factores de vulnerabilidad.

\section{La ciudad de Salta y su proceso de expansión}

La fundación de la ciudad de Salta se remonta al año 1582, cuando Hernando de Lerma, por mandato del virrey Toledo, la llamó Ciudad de San Felipe del Valle de Lerma. Su emplazamiento se relaciona con el lugar estratégico que ocupaba para asegurar la ruta entre el Alto Perú y el Río de la Plata ${ }^{3}$.
3. Si bien el lugar elegido para su fundación era estratégico en términos de potencialidad comercial, su emplazamiento resultaba inapropiado para el asentamiento humano, en virtud de que se la situó en una depresión del terreno que la rodea. Las consecuencias actuales son las siguientes: polución ambiental, distribución irracional de sus espacios y zonas altamente inundables, entre otras (CANCINOS, 2002). 
4. Los lugares de invernada de las mulas se ubicaban en puntos muy cercanos a la ciudad.

5. Los migrantes en Argentina se empleaban como asalariados con remuneraciones que en el Litoral duplicaban los salarios medios de Europa. En el caso de Salta, se mantenían relaciones semiserviles o formas de trabajo forzado, como la institución del conchabo, sobre todo dentro de las fincas que regenteaban los grupos de la aristocracia criolla. Esta situación fue una de las causas más importantes de la escasa migración de ultramar en esta región (ÁLVAREZ

LEGUIZAMÓN, 2004).

6. La tasa de crecimiento intercensal pasó de un 23,6 $\%$ en el período 1914- 1947 a un $43 \%$ entre 1947-1960

(AgUILAR y SBROCCO, 2009).
Durante el período colonial, la ciudad se desarrolló como centro mercantil para la compra-venta de ganado, ya que se ubicaba sobre los caminos que conectaban a las "provincias del sur" con los mercados altoperuanos. Funcionaba como una estación de tránsito con sus ferias de mulas y campos de invernada ${ }^{4}$. También actuaba como centro consumidor y reexportador de efectos de Castilla introducidos desde Buenos Aires y de tucuyos ingresados desde el Alto Perú (MATA, 1996). Estas actividades trajeron prosperidad económica en las últimas décadas del siglo XVIII e incrementaron significativamente la población debido al aporte de inmigrantes españoles e indígenas provenientes del Alto Perú.

Con posterioridad a las guerras de la independencia, Salta dejó de ser una región de tránsito en el espacio mercantil andino para convertirse en una provincia de frontera de la naciente República Argentina. La próspera economía del período colonial como resultado de esta nueva configuración entró en un camino de retracción (Justiniano, 2008). Pasó a funcionar, junto al resto de las provincias del NOA, como periferia del mercado del Litoral vinculado con el Atlántico y como periferia del nuevo espacio económico andino resultante de este proceso.

En el último cuarto del siglo XIX se asiste a una declinación de la economía provincial y una reorientación de todo el NOA hacia Buenos Aires (Justiniano, 2008). Perdió importancia el comercio mular, mientras que la ganadería y las curtiembres se mantuvieron entre las principales actividades. Las colocaciones de suela en el litoral, a diferencia del período anterior, pasaron a ser la mayor fuente de ingreso provincial.

En este período, también, comienzan a desarrollarse algunos cultivos industriales (caña de azúcar, tabaco y vid), cuya expansión es favorecida por la llegada del ferrocarril (ACRECHE ET AL., 2011). A la actividad azucarera, pese a la importancia que adquirió, no se la consideraba como fuente de riqueza en la economía provincial, en tanto recién fue gravada en 1905.

Estos vaivenes de la economía provincial no modificaron sustancialmente el desarrollo de la ciudad de Salta, que se mantuvo sin grandes cambios hasta la década de $1930^{5}$. Recién entre 1940-1960 registra una visible expansión de la trama urbana, con un fuerte crecimiento de su población ${ }^{6}$. ÁLVAREZ LEGUIZAmón (2004) sostiene que este período se caracteriza por la constitución de un mercado de trabajo libre, la relajación de las relaciones semiserviles junto con la ampliación de grupos asalariados y la conformación de una incipiente clase media. 
El crecimiento se produjo, principalmente, por la migración de importantes contingentes desde el interior de la provincia a la ciudad, que pudieron acceder a loteos económicos y construyeron sus viviendas mayoritariamente por autoconstrucción (Aguilar ET AL., 2000) ${ }^{7}$. Los sectores medios en ascenso también accedieron a la compra en loteos mejor localizados y a la construcción por encargo. Estos movimientos se vinculan con la ampliación de los servicios urbanos y con el crecimiento del sector asalariado en el mercado de trabajo local.

Cabe destacar que la modalidad de ocupación del suelo a través de los loteos, entre los años 1940 y 1974, significó un anexo de 1200 ha de tierras en el área adyacente al casco más antiguo de la ciudad (Aguilar et AL., 2000). SBrocco (1999) destaca en este período el rol central del sector inmobiliario en la urbanización de miles de hectáreas.

Entre las décadas de 1960 y 1980 tuvo lugar una segunda ampliación de la ciudad como resultado de una transformación de la estructura social en cuanto a sus características demográficas. Desde 1960, hay una expansión urbana por el propio crecimiento vegetativo de la población y, principalmente, por el impacto de la migración de origen rural hacia la ciudad capital $^{8}$. La oferta de loteos continuó hasta mediados de 1970, aunque ya en menor escala, y desde esta década también se destaca la intervención del Estado con políticas habitacionales fuertemente centralizadas que dieron lugar a lo que se denomina periferia estatal: la localización de megabarrios en los extremos norte y sur de la ciudad. Estas viviendas estaban destinadas a sectores medios asalariados. A mediados de los 80 se desarrollan, además, programas de viviendas que atendieron parcialmente la demanda de sectores de escasos recursos (Agullar y SBRocco, 1997). Surgen también "villas de emergencia” en los alrededores de áreas urbanas consolidadas.

El tercer momento de expansión se produce a partir de la década de 1990. Se caracteriza por la difusión de asentamientos informales, que tienen como protagonistas a pobladores jóvenes excluidos del mercado de trabajo, la suburbanización según el patrón de "desconcentración” a través de barrios cerrados y la extensión hacia municipios vecinos de la ciudad capital, como Vaqueros (Norte) y Cerrillos (Sur) ${ }^{9}$.

El acceso al crédito y ahorros propios de sectores medios y altos junto a la oferta de tierras, la mejora de la infraestructura, el auge de la tecnología de la información son elementos que influyeron en este nuevo patrón de crecimiento (Aguilar y SвROcco, 2009, p. 163).
7. Los loteos económicos son barrios que surgieron a partir de la subdivisión masiva de tierras de uso ruraly que se vendieron en cuotas en un plazo de entre dos y cinco años (AgUILAR y SBROCCO, 1997).

8. Se produce también un acelerado proceso de urbanización de la provincia.

9. También se registra una expansión de estas urbanizaciones hacia el municipio de Villa San Lorenzo (oeste), pero este todavía no está anexado al aglomerado. 
10. Vaqueros, hasta las décadas de 1930-1940, prácticamente era una sola finca destinada a la producción agropecuaria. Recién en este período llegaron varias familias de origen europeo, particularmente españoles, que arrendaron tierras para cultivo de verduras, primero, y luego de tabaco. A partir de 1946, con la llegada del peronismo al poder, comien-

za un lento movimiento de transformación en las carac-

terísticas de la estructura productiva y en el proceso de asalarización. El municipio de Vaqueros como jurisdicción político-administrativa fue fundado en 1970 (AgUILAR y CostilLA, 2009).

11. Se llamaba pisteros a quienes el patrón cedía en préstamo una parcela de tierra dentro de la finca, tener animales y una huerta para subsistencia.

Figura 1. Aglomerado Gran Salta. 2010. División por localidades
Este proceso de expansión urbana, como ya se mencionó, implicó una conurbanización con otras localidades, pertenecientes a distintos departamentos de la provincia, que conformó el aglomerado Gran Salta (ver figura 1). En el caso de Vaqueros (departamento La Caldera), la contigüidad remite a la década de 1970, luego de que asistiera a un movimiento de población asociado a la fuerza de trabajo que se incorpora a la producción tabacalera ${ }^{10}$.

Si bien se trataba de migración golondrina, muchos de ellos se instalaron de manera definitiva, asentándose precariamente dentro del predio de las fincas en calidad de piseros. Con posterioridad varios de ellos accedieron a la compra en el primer loteo, dando origen a lo que hoy es la zona más densamente poblada e identificada como el centro de Vaqueros (Aguilar y Costilla, 2009, p. 59) ${ }^{11}$.

Como parte de este proceso se funda el municipio de Vaqueros como jurisdicción político-administrativa.

El sur la ciudad de Salta se expandió hacia el municipio de Cerrillos - la zona de anexión es villa Los Álamos, el barrio con mayor antigüedad y uno de los más poblados-. Cerrillos es cabecera del departamento del mismo nombre que incluye las localidades de La Merced, San Agustín y parajes como Colón, La Isla, Sumalao y Las Pircas.
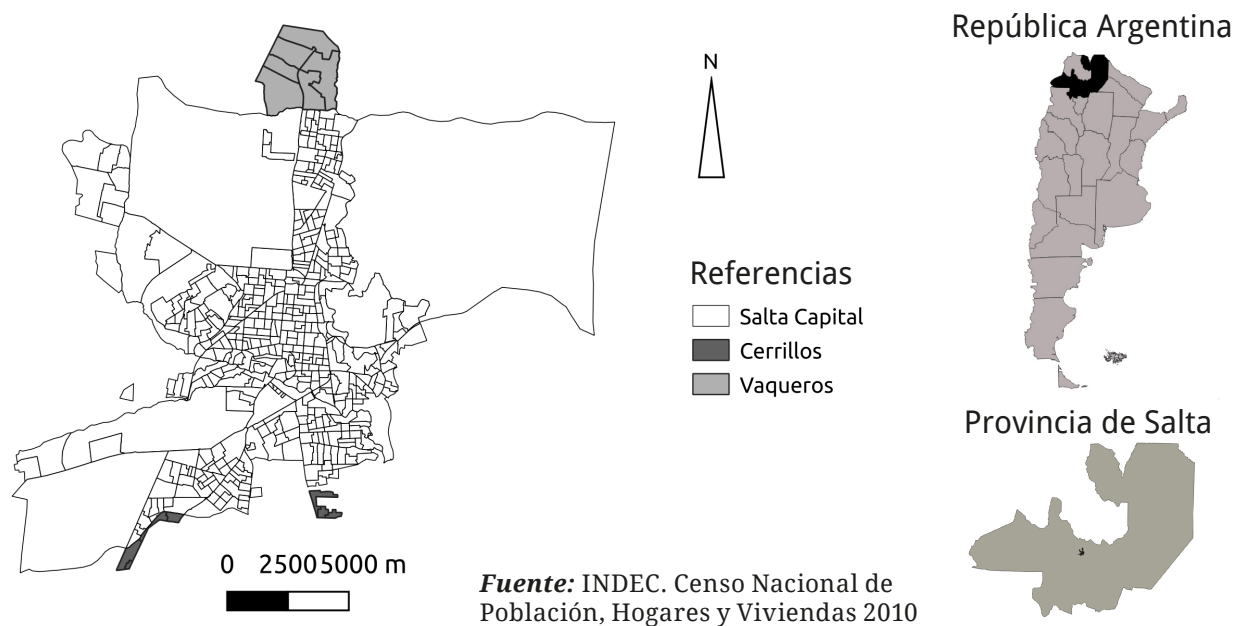

Provincia de Salta

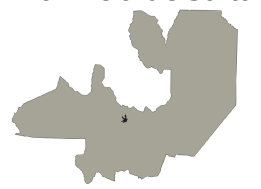




\section{Proceso de urbanización de la pobreza en Gran Salta}

La urbanización de la pobreza en Gran Salta, como en la región, reconoce más de un siglo de desarrollo, y fue adquiriendo matices espaciales, como se expone en este apartado, conforme la disposición en la ciudad de las poblaciones pobres. Se inicia de manera lenta, a principios del siglo XX, con la asalarización de campesinos y siervos que, a partir de la pérdida de posesión de los medios de producción o la ruptura de relaciones serviles, migran a la ciudad para incorporarse al incipiente mercado de trabajo. Estos se asientan en ranchos o viviendas obreras situadas en la periferia. De hecho, una ordenanza municipal de la época (26/10/1911) establece un radio de exclusión para su instalación. Álvarez LeguizAmón ET AL. sostienen al respecto que

ciudades capitales como Salta pasan de ser consideradas aldeas inurbanas semi rurales donde habitaban las élites aristocráticas y sus siervos, a ciudades que paulatinamente se van vistiendo de ropaje higiénico y civilizatorio, integrando al campesino pobre, al trabajador y al artesano de una manera subordinada (2010, p. 71).

Si bien con las prácticas higienistas se promovía el agua corriente, la vivienda salubre y la vacunación, estas no llegaban a los pobres. La población seguía fuertemente afectada por enfermedades endémicas, como la tuberculosis y el mal de Chagas, y cerca de la tercera parte de las familias de la ciudad vivían hacinadas (Á́lvarez LeguizAmón et AL., 2010). A ello hay que agregar los altos porcentajes de analfabetismo y la carencia de regulaciones del trabajo, condiciones que dan cuenta de una situación social crítica para los trabajadores.

Hasta 1930, el crecimiento demográfico y espacial de la ciudad fue lento, respondiendo a este esquema de segregación urbana que conllevó la división en dos Saltas: la del centro y la Salta de los arrabales (Соввасно у ADEт, 2002). Mantenía las características de una pequeña ciudad de provincia. No obstante, la sucinta pero continua migración interna de campesinos fue ampliando su base social.

Además de crecer los problemas habitacionales en la periferia urbana, los migrantes se instalaron en conventillos y, en menor medida, pensiones ubicadas en la zona céntrica de la ciudad, donde las condiciones eran de extrema precariedad:

Deficiencias higiénicas, falta de servicios sanitarios, de instalación eléctrica [...] de piso, pintura en las habitaciones y revoque en las cocinas. Por la carencia de 
12. En el caso de San Miguel de Tucumán, los conventillos $e$ inquilinatos fueron desapareciendo o evolucionando hacia una nueva tipología constructiva previo desalojo de sus habitantes (NATERA RIVAS, 1996).

13. Entre 1930 y 1945 se produce una ruptura a partir de la relajación progresiva de las relaciones semiserviles $y$ de las leyes de conchabo. Hasta ese momento, la aristocracia salteña buscó retener sus espacios de dominio privado y uso de la violencia física en la finca y también en la casa con los criados y conchabados (ÁLVAREZ LEGUIZAMÓN ET AL., 2010).

14. La mayoría de los barrios estuvo entre cuatro y treinta y cuatro años sin infraestructura básica - agua, cloacas y luz-, y aún existen barrios completos sin cloacas y otros con instalación parcial. Debido a los escasos ingresos de la población, los pozos ciegos existentes son muy precarios, factor que incide en la contaminación de las napas subterráneas (AgUILAR ET AL., 2000). servicios sanitarios las aguas servidas son derramadas hacia la calzada, constituyendo un peligro para la salud pública dada las emanaciones pestilentes que se desprenden (Boletín Municipal, noviembre de 1941).

Los habitantes de los conventillos tenían diversas ocupaciones: choferes, costureras, aurigas, trabajadores asalariados, zapateros, vendedores ambulantes, prostitutas, algunas mujeres solas, viudas o abandonadas con varios hijos, que se ganaban el sustento con el lavado y planchado en casas cercanas. Las pensiones reunían mejores condiciones y eran habitadas por familias, hombres solos, comerciantes y artesanos con modestos talleres (CoRвАСно У ADET, 2002). Si bien no se encontraron estudios sobre la evolución de esta solución habitacional, es presumible que hayan sucumbido en función del alza de los precios del suelo urbano y las exigencias de mejoras, a través de leyes, a partir de la década de 1930, como ocurrió en otras ciudades ${ }^{12}$.

Las acciones del Estado para mejorar las condiciones de las viviendas populares fueron escasas. El único caso, antes del gobierno peronista, fue el realizado durante la intendencia de Ceferino Velarde, entre los años 1936-1940, con la construcción del primer barrio obrero, que contó con sesenta viviendas y mercado municipal propio (СоRвACHO y ADET, 2002).

A partir de 1947 se registra un significativo aumento poblacional que se produce tanto por el crecimiento vegetativo como por el aporte de migrantes internos. Como se expuso en el apartado anterior, recién en este momento (entre 1940 y 1960) adquiere fisonomía el mercado de trabajo libre ${ }^{13}$. Gran parte de los migrantes y sectores populares urbanos pudieron acceder a loteos urbanos económicos, por la modalidad de comercialización a través de cuotas, y a la vivienda por autoconstrucción (AgULLAR ET AL., 2000).

Si bien estos sectores con los loteos resolvieron el problema del acceso al suelo y tenencia legal, debieron afrontar condiciones de precariedad habitacional. Las mejoras relativas alcanzadas no fueron resultado de las "bondades de la urbanización" o de la acción espontánea del Estado, sino de prácticas y estrategias de sus primeros habitantes y de ciertas organizaciones, principalmente centros vecinales, que surgieron producto de esas acciones ${ }^{14}$.

El orden de demandas, gestión y posterior provisión de la infraestructura en los barrios de loteos, se fue realizando dando prioridad, a las necesidades más vitales. 


\section{Urbanización de la pobreza en el aglomerado Gran Salta (Noroeste Argentino).}

Sus manifestaciones actuales

Primero: luz, agua y alumbrado público incandescente; en segundo lugar, cloacas y, por último: gas y pavimento (Agullar ET AL., 2000, p. 145) ${ }^{15}$.

Varios de los problemas ambientales de los terrenos loteados, como su localización en zonas inundables o sobre zanjones y canales, continúan sin solución en la actualidad (AgulLAR ET AL., 2000).

Desde 1960, la migración de origen rural hacia la ciudad capital se intensifica, conjuntamente con un acelerado proceso de urbanización de la provincia. La ciudad ya no pudo absorber a esta nueva población, y se producen pequeñas ocupaciones de tierra en los alrededores de áreas urbanas consolidadas, conocidas como "Villas de Emergencia” (Sвrocco, 1999).

El Estado provincial, como ya se expuso, en la década de 1970 construyó conjuntos habitacionales en las afueras de la ciudad, destinados a sectores medios asalariados $^{16}$. Estos, sin embargo, por su lejanía, no contaban con infraestructura ni servicios básicos, como el transporte público.

Durante la década de 1990, se asiste a una nueva etapa del proceso de urbanización de la pobreza, con la multiplicación de asentamientos en diferentes zonas de la ciudad, principalmente en el sureste. Estos se forman a partir de la ocupación organizada de tierras fiscales o privadas por sectores de población, jóvenes en general, excluidos del mercado de trabajo o insertos de forma muy inestable, quienes luego reclaman al Estado el derecho a la compra a precios accesibles (Aguilar y Costilla, 2009). Surgieron alrededor de 87 "barrios informales", muchos de los cuales se radicaron en lugares de peligrosas condiciones de habitabilidad, fuertes pendientes o bajos, inundables, con escasa o precaria infraestructura (GómEz, 2017) ${ }^{17}$.

En contraste - en el otro extremo de la pirámide social, divididos y sin integrarse con los sectores vecinos- los sectores de clase alta y media-alta ocupan la periferia, pero generalmente en lugares altos o de buenos paisajes y visuales. "Son los barrios privados, urbanizaciones cerradas, clubes de campo o countries, encerrados con una moderna versión de muralla elitista que marca el adentro y el afuera,
15. El área con menor provisión de servicios urbanos básicos es la que rodea la zona de crecimiento por loteos. Esta condición contrasta con mejores provistas a áreas de ocupación más reciente habitadas por sectores medios y altos (AGUILAR ET AL., 2000).

16. Los límites de la ciudad se modifican rotundamente con la construcción del barrio Santa Ana o el barrio Ciudad del Milagro, por ejemplo, durante los años 80, en las márgenes sur y norte respectivamente, $y$ se extienden exponencialmente en un corto tiempo las fronteras de la ciudad de manera discontinua y con llamativos espacios vírgenes intersticiales (AgulLAR, 1987).

17. GÓMEZ (2017) considera que la denominación "barrios informales" es un eufemismo que pretende eludir elípticamente la denominación asentamientos de miseria y la inequidad. El autor menciona los asentamientos Convivencia, Fraternidad, Gauchito Gil, Villa Juanita, San Juan de Dios y La Paz, en el costado sudeste de la ciudad, los que se caracterizan por una extrema precariedad. Hacia el oeste, los nuevos asentamientos se ubicarían por detrás de Siglo XXI y Solidaridad, como San Silvestre, Bicentenario, Virgen del Rosario. Al sudeste surgieron El Cambio, Floresta, Canillitas, Finca Torino, San Lucas, San Mateo, Las Colinas y Autódromo; mientras que al nordeste menciona a los asentamientos 15 de Septiembre, La Unión, Villa Lata, Divino Niño y 1 de Mayo. 
18. A las urbanizaciones cerradas El Tipal y la Almudena, al noroeste de la ciudad, surgidas en el año 2000 aproximadamente, le siguieron La Lucinda al sureste, Valle Escondido, Altos de San Bernardo al este; El Portal de Limache, El Aybal, El Prado y Ogimoro al suroeste, respectivamente

(GÓMEZ, 2017).

19. Este proceso implicó el incremento del precio de la tierra y la expulsión de nuevas generaciones de vaquereños hacia los asentamientos ubicados en las márgenes del río Vaqueros en el departamento Capital (AgUILAR y SBROCCO, 2009). generalmente próximos a las vías de circulación y las autopistas de reciente trazado" (GóMEZ, 2017, p. 387) ${ }^{18}$. Se menciona esta modalidad de ocupación en tanto su irrupción implicó la acentuación de los contrastes sociales y un proceso de acumulación de desventajas para las poblaciones que viven en áreas de pobreza, asociado a la intensificación de la segregación socioespacial y la fragmentación urbana.

\section{Distribución actual de la población en Gran Salta según el ICVI}

La aplicación del ICVI en 2001 (ver figura 2) refleja que en el área central, coincidente con el centro financiero y comercial y la zona residencial más antigua y consolidada de la ciudad, se concentra la población con mejores condiciones de vida, mientras que en un gradiente hacia el oeste y este se localizan las poblaciones con mayores privaciones. Las áreas periféricas en los extremos oeste y este, dentro de esta gradiente, son las que se encuentran en peor situación.

Se observa también que los radios censales de la capital próximos al límite con Vaqueros al noroeste - concentran población con condiciones de vida precarias. Estos se corresponden con los barrios 15 de Setiembre, Juan Manuel de Rosas, La Tradición, Patricia Heitman, 17 de Octubre, La Unión y Balneario.

Los radios que pertenecen a Vaqueros se ubican en el cuartil III, y su población está integrada por trabajadores del tabaco que en la década de 1960 accedieron a loteos de viviendas unifamiliares. En su mayoría eran migrantes golondrinas provenientes del interior de la provincia y de Bolivia que se asentaron en el lugar. Residen también antiguos pobladores que, como ya se expuso, habían accedido a las tierras en calidad de piseros. Con posterioridad, principalmente en la década del 90, los loteos en el resto de la localidad se destinan a sectores medios y medios-altos ${ }^{19}$.

En el extremo sureste se localiza también población con altos niveles de privación. Esta área constituye una de las de mayor expansión en la ciudad, como resultado de la localización de numerosos asentamientos (algunos de los cuales ya se consolidaron como barrios): Solidaridad, Fraternidad, Norte Grande, San Ignacio, Floresta, 20 de junio, Juanita, La Fama, El Milagro, El Jardín, El Manjón, Alta Tensión, El Cóndor, Primera Junta, La Paz, Las Colinas, San Lucas, Canillita y San Mateo. 


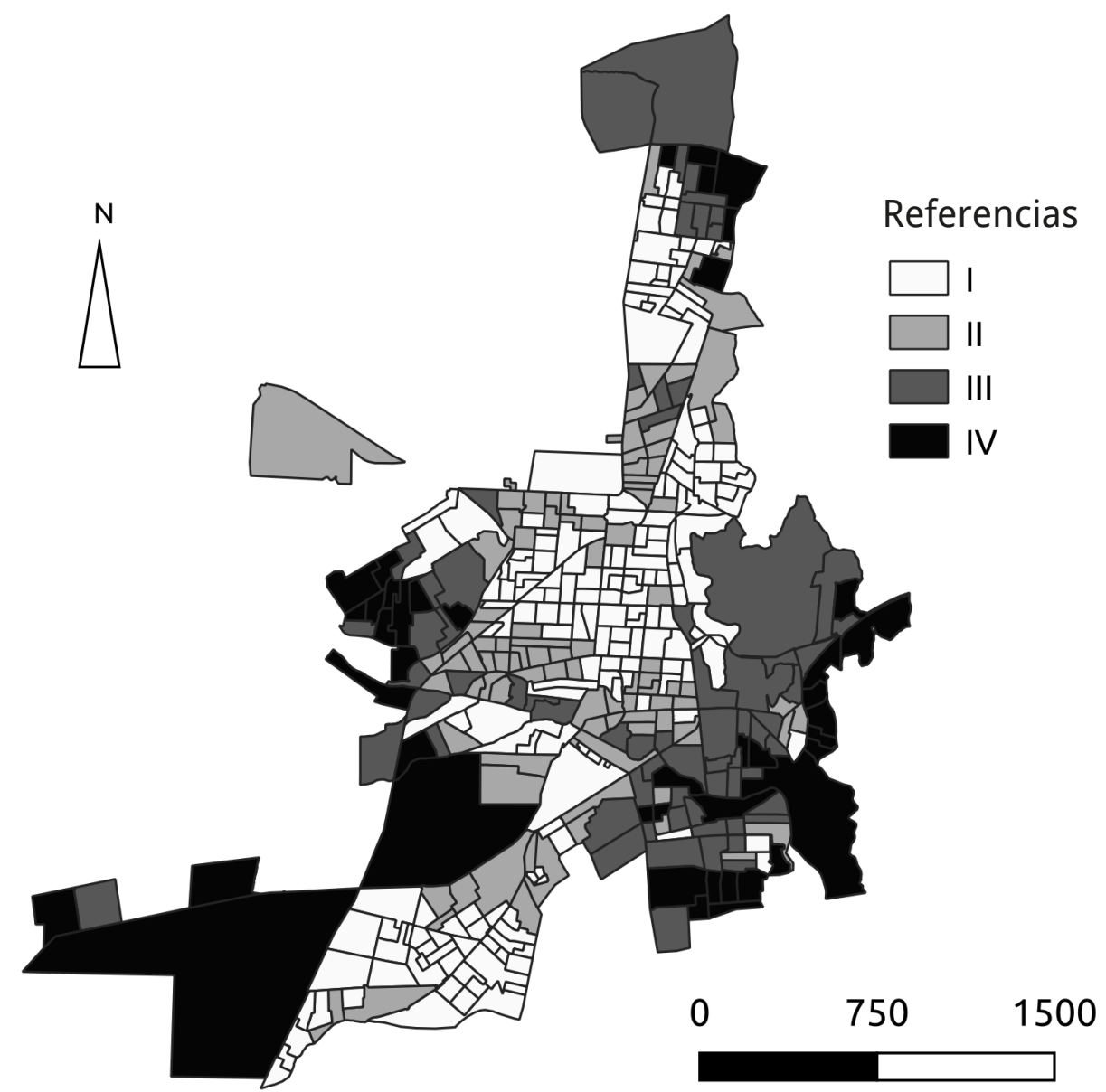

Fuente: Censo Nacional de Población, Hogares y Viviendas 2001

Figura 2. Gran Salta. Índice de Condiciones de Vida, 2001 
20. Se trata de un programa provincial destinado a jóvenes de entre 18 y 35 años.

21. Ver: https://informatesalta.com.ar/contenido/40684/ lomas-de-medeiros-los-verdaderos-perjudicados; $h$ ttps://cuartopodersalta.com. ar/las-casas-se-siguen-entregando-a-dedo/
La aplicación del ICVI para el año 2010 (ver figura 3) no resulta comparable espacialmente con el año 2001, puesto que la morfología del aglomerado, así como la cantidad, tamaño y diseño de sus radios censales, no es igual. No obstante, pueden observarse las áreas de crecimiento y las tendencias en la distribución socio-económica de la población, en virtud de la inclusión de nuevos radios censales.

El área de expansión noroeste comprende nuevos radios de la localidad de Vaqueros que se ubican el cuartil III. Incluye, también, un complejo habitacional, denominado El Huayco, construido por el instituto de la vivienda provincial en terrenos de propiedad del Ejército argentino, para sectores de clase media. Hasta 2010 se encontraba habitada solo una parte, que en el mapa coincide con el radio del cuartil I. Posteriormente, se construyeron distintas ampliaciones que ya están pobladas y otra urbanización denominada Mirasoles. Como parte de la urbanización, se edificaron monoambientes destinados a jóvenes en el marco del Programa "Mi primera vivienda" 20.

Los radios correspondientes al cuartil I que se ubican en el área mencionada (noroeste) comprenden urbanizaciones cerradas, como La Almudena y El Tipal, y los barrios Los Pinos I y II. También se encuentra la urbanización estatal Lomas de Medeiros, donde la adjudicación a los beneficiarios se realizó - aunque no eran sus destinatarios-a personas con alto poder adquisitivo ${ }^{21}$.

El área de expansión nordeste presenta una particularidad. Si bien los nuevos radios se corresponden al cuartil III, se trata de una zona de desarrollo de grandes urbanizaciones cerradas. Allí actualmente se localizan los barrios privados Valle Escondido, La Lucinda I y La Lucinda II. En 2010, año de realización del censo, los dos primeros se encontraban en construcción, mientras que el último es posterior. Se trata de emprendimientos en espacios de alto valor paisajístico.

En el suroeste los radios nuevos corresponden a las urbanizaciones cerradas El Prado, Ojimoro y El Aybal. Se sitúan también distintas urbanizaciones en proceso de consolidación, como Vía Aurelia, Estación Alvarado y La Querencia. En este sector del aglomerado se ubica la localidad de San Luis, caracterizada por casas de estilo campestre que suelen alquilarse para veraneo, tal como es el caso de villa Rebeca. 
El sector sureste continúa recepcionando a los sectores peor posicionados en la estructura social. Surgieron numerosos barrios: San Alfonso, Gauchito Gil, Sanidad, Finca Valdivia, El Círculo y El Palenque. Por otra parte, se anexa villa Los Álamos, que es el barrio más antiguo de la localidad de Cerrillos.

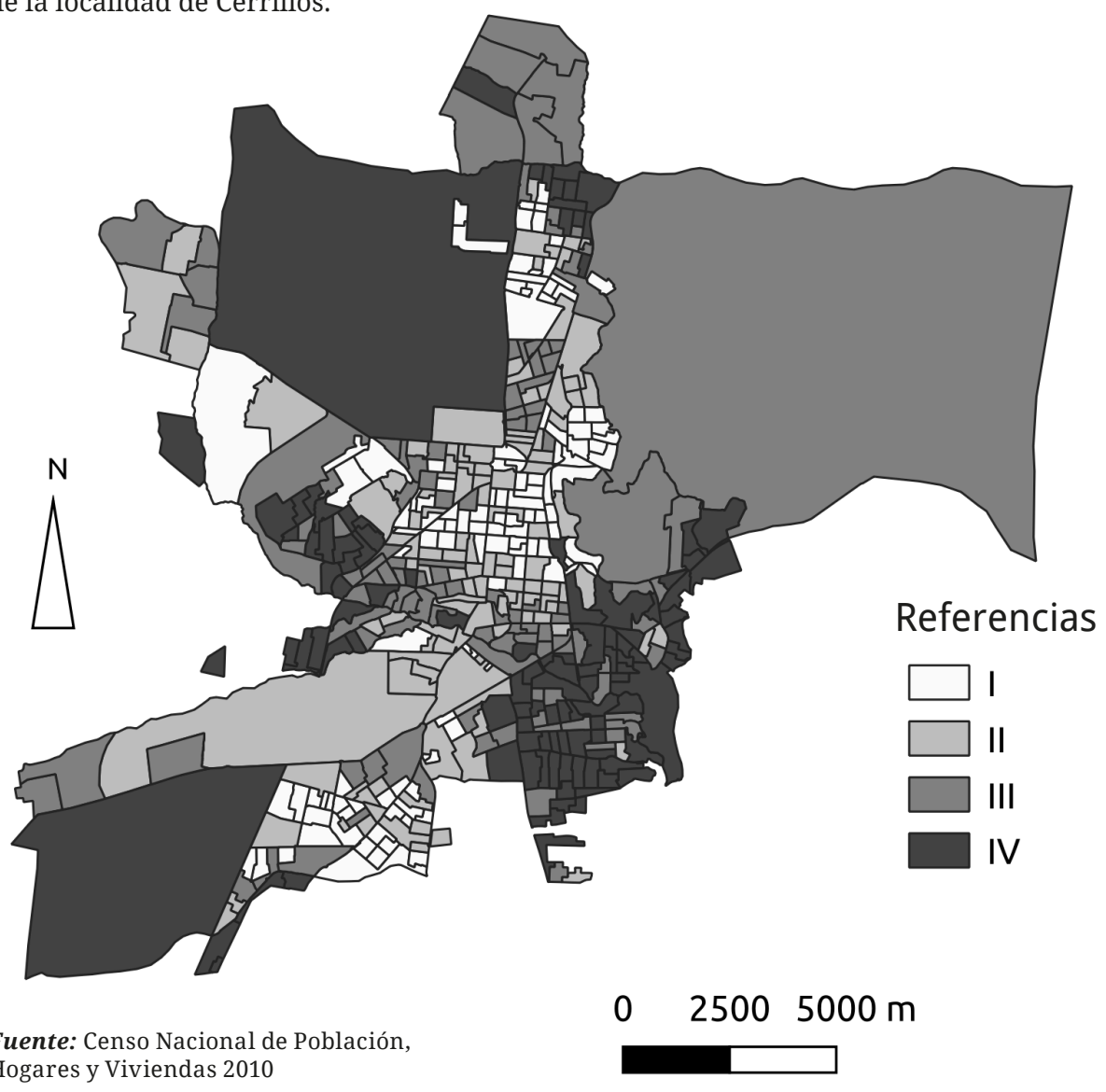

Figura 3. Gran Salta. Índice de Condiciones de Hogares y Viviendas 2010

Vida, 2010 


\section{Estructura demográfica y condiciones de vida por cuartiles}

Las tablas 1 y 2 muestran la distribución de la población según el ICVI, utilizando cuartiles, para los años 2001-2010. De ellas se desprende que tanto la población como los hogares situados en el cuartil I, los mejor posicionados en la estructura social, decrecieron un 4,3 $\%$ y un $6 \%$, respectivamente. Asimismo, aumentan los hogares y población situados en el cuartil II, lo cual refleja una redistribución del primer cuartil hacia dicho nivel.

El peso del cuartil IV aumenta en 2010, mientras que baja la proporción del cuartil III. Esta redistribución refleja un deterioro de las condiciones de vida en términos de educación y vivienda. Centrándonos solo en el cuartil IV, es clara la incorporación de un mayor número de personas y hogares en el universo de la pobreza, medida en esta instancia a partir de un índice de condiciones de vida. Se observa con claridad que 39.868 personas son incluidas en este cuartil, junto a 8016 nuevos hogares incorporados en este estado de carencias.

Tabla 1

\begin{tabular}{|c|c|c|c|c|}
\hline \multicolumn{5}{|c|}{ Índice de Condiciones de Vida. Año 2001} \\
\hline Cuartiles & Población & Hogares & \% Población & \% Hogares \\
\hline I & 115394 & 36680 & 24,2 & 28,7 \\
\hline II & 95717 & 27149 & 20,1 & 21,2 \\
\hline III & 140905 & 35065 & 29,6 & 27,4 \\
\hline IV & 123935 & 28989 & 26 & 22,7 \\
\hline Total & 475951 & 110032 & 100 & 100 \\
\hline
\end{tabular}

Tabla 2

Fuente. Censo Nacional de Población, Hogares y Viviendas 2001

\begin{tabular}{|c|c|c|c|c|}
\hline Cuartiles & Población & Hogares & \% Población & \% Hogares \\
\hline I & 108926 & 31804 & 19,9 & 22,7 \\
\hline II & 127604 & 34847 & 23,3 & 24,8 \\
\hline III & 147817 & 36586 & 27 & 26,1 \\
\hline IV & 163803 & 37005 & 29,9 & 26,4 \\
\hline Total & 548150 & 140242 & 100 & 100 \\
\hline
\end{tabular}




\section{Características sociodemográficas de la población en condiciones de pobreza}

El análisis sobre las características sociodemográficas de las áreas de mayor pobreza se realiza a partir de la selección del cuartil IV, y se comparan los valores hallados con los guarismos de Gran Salta.

La población que reside en dichas áreas representa el 29,93 \% del total del aglomerado, abarcando a 163.803 personas. Estas habitan 37.005 hogares, lo que da un promedio de 4,4 personas por hogar, siendo superior al tamaño de los hogares del Gran Salta (3,9 personas por hogar).

Las áreas de pobreza presentan una estructura de población joven, que se evidencia en una pirámide poblacional de base ancha con fuerte peso de la población infantil y de jóvenes de hasta diecinueve años principalmente (ver figuras 4 y 5). Este segmento poblacional conforma el 47,6 \% de la población total, mientras que en el aglomerado representa el 38 \%. En consonancia con ello, se presume que la población de estas áreas registra mayores tasas de natalidad y fecundidad y de fecundidad adolescente. Se observa también una significativa mortalidad/emigración en grupos juveniles a partir de los veinticinco años, en los cuales la composición se asemeja a la del aglomerado.
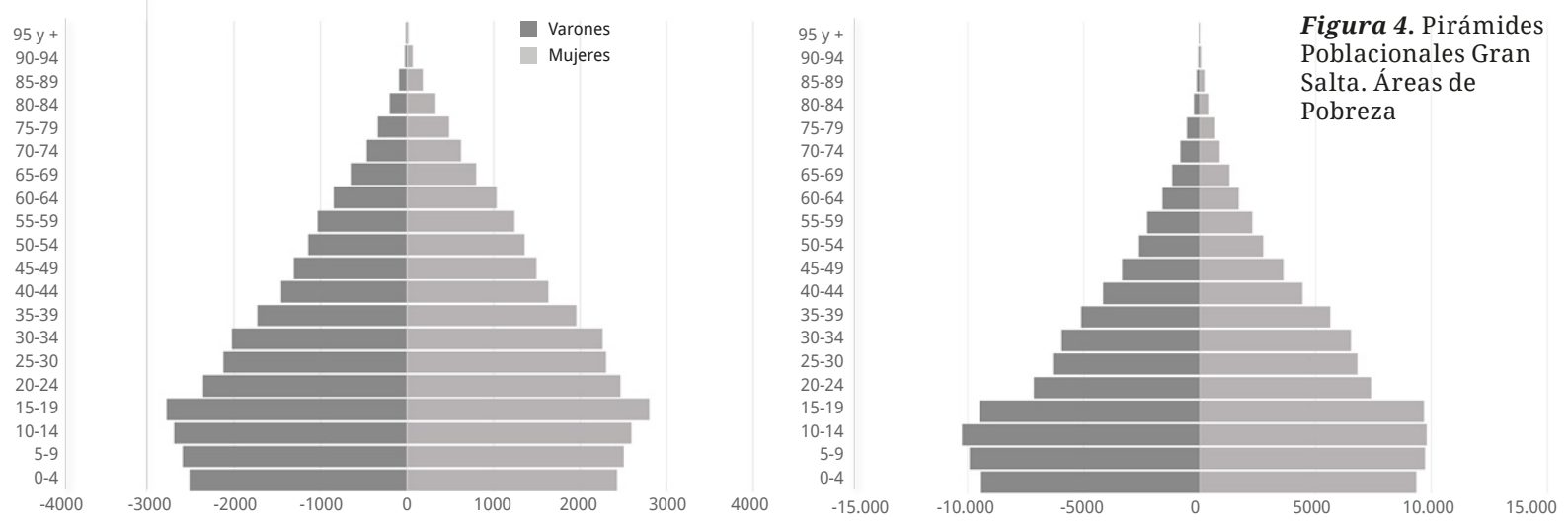

Fuente: Censo Nacional de Población, Hogares y Viviendas 2010 
El nivel educativo de los/las jefes/as de hogar en las áreas de pobreza es considerablemente inferior al del conjunto del aglomerado. Mientras que el 31,4 completó el nivel primario, la terminalidad en el secundario se reduce a casi la mitad, y el acceso al nivel superior es exiguo (ver tabla 3). Es llamativo que aproximadamente el $18 \%$ no terminó la primaria.

Tabla 3

\begin{tabular}{lcc}
\hline \multicolumn{3}{l}{ Nivel educativo de los/las jefes/as de hogar } \\
\hline & Gran Salta & Áreas de pobreza \\
\hline COMPLETÓ Primario & 21,8 & 31,4 \\
\hline EGB & 0,1 & 0,4 \\
\hline Secundario & 22,1 & 17,6 \\
\hline Polimodal & 0,3 & 0,5 \\
\hline Superior no universitario & 6,0 & 2,4 \\
\hline Universitario & 7,9 & 1,3 \\
\hline Post universitario & 0,7 & 0,1 \\
\hline Subtotal Completó nivel & 58,9 & 53,7 \\
\hline NO COMPLETÓ Primario & 10,4 & 17,7 \\
\hline EGB & 0,4 & 0,8 \\
\hline Secundario & 17,5 & 21,7 \\
\hline Polimodal & 0,5 & 1,0 \\
\hline Superior no universitario & 3,7 & 2,6 \\
\hline Universitario & 8,4 & 2,4 \\
\hline Post universitario & 0,3 & 0,1 \\
\hline Subtotal No Completó nivel & 41,1 & 46,3 \\
\hline TOTAL & $\mathbf{1 0 0}$ & $\mathbf{1 0 0}$
\end{tabular}

Fuente: Censo Nacional de Población, Hogares y Viviendas 2010

En relación con la participación en el mercado de trabajo, se observan niveles de ocupación y desocupación similares al aglomerado (60 \% y 5 \%, respectivamente) (ver tabla 4). La diferencia probablemente está en el tipo de calificación y el nivel de formalidad. 


\section{Tabla 4}

\begin{tabular}{lcc}
\hline Condición de actividad & & \\
\hline & Gran Salta & Áreas de pobreza \\
\hline Ocupado & 60,9 & 60 \\
\hline Desocupado & 5,2 & 5,4 \\
\hline Inactivo & 33,9 & 34,6 \\
Total & $\mathbf{1 0 0}$ & $\mathbf{1 0 0}$
\end{tabular}

Fuente. Censo Nacional de Población, Hogares y Viviendas 2010

\section{Conclusiones}

En el presente trabajo, las manifestaciones actuales de la pobreza urbana en el aglomerado Gran Salta se explican desde una perspectiva histórica teniendo en cuenta las transformaciones económicas, sociales y territoriales. Si bien se asiste a un aumento de las poblaciones afectadas en las últimas cuatro décadas y a una acumulación de desventajas, estos aspectos se relacionan con la conformación y el devenir del mercado de trabajo y el propio proceso de urbanización de la ciudad.

El análisis realizado permite dividir el proceso de urbanización de la pobreza en Gran Salta en tres momentos. El primero, que se sitúa entre inicios del siglo XX y la década de 1950, transcurre desde la incipiente asalarización de campesinos hasta la conformación de un mercado de trabajo libre. En este se identifican ciertas privaciones habitacionales y problemas de empleo que atraviesa la población rural que llega a la ciudad, pero que todavía no adquieren un carácter disruptivo.

Se distinguen ciertas intervenciones del Estado dirigidas a modificar dichas condiciones, como las prácticas higienistas y luego las políticas de ampliación de derechos sociales durante el peronismo. A partir de la década de 1940, gran parte de los migrantes pudieron acceder a loteos urbanos económicos y, mediante la autoconstrucción, a la vivienda, aunque fuese en condiciones de precariedad habitacional. 
El segundo momento se desarrolla entre 1960-1980, cuando se produce un fuerte proceso migratorio desde las áreas rurales de la provincia de Salta a la capital. Este proceso tiene mayor intensidad durante la primera década, y provocó la aparición de las primeras villas de emergencia en los alrededores de áreas urbanas consolidadas. Si bien la oferta de loteos económicos se extendió hasta 1974, un sector no pudo acceder y recurrió a esta salida habitacional. Se asiste también a la construcción de la llamada periferia estatal, que si bien estuvo dirigida a sectores medios, las condiciones de entrega de las viviendas generaron precariedad habitacional. En este caso el propio Estado actuó directamente como productor de condiciones de pobreza.

El tercer momento, que tiene lugar desde la década de 1990, se caracteriza por el crecimiento sostenido de la pobreza y un proceso de acumulación de desventajas para las poblaciones afectadas, especialmente en las que esta tiene un carácter persistente. Se afianza de esta manera un modo de vivir la ciudad con una desigualdad que persiste en el tiempo - como contracara se desarrollan áreas de opulencia-y genera una marcada fragmentación urbana y nuevas expresiones de la segregación socioespacial.

La aplicación del ICVI, en el período 2001-2010, permitió analizar la distribución de la población en el aglomerado identificando las tendencias al crecimiento y consolidación de áreas de riqueza, con la difusión de numerosas urbanizaciones cerradas, y su opuesto, de áreas de pobreza. Estas últimas, a diferencia de las primeras, que avanzan sobre distintas zonas de la periferia de la ciudad - las de mayor calidad ambiental—, tienden a concentrarse en los lugares ya caracterizados por los altos niveles de privación. En este sentido, se observa la cristalización de la pobreza en determinadas áreas - barrios de la capital situados en el límite noroeste con Vaqueros y en el extremo oeste-, más allá de algunas mejoras a nivel de servicios e infraestructura. Lo que ocurre en la zona suroeste es la máxima expresión de dicho proceso, ya que en los últimos treinta años pasó de ser una zona totalmente deshabitada a convertirse en la de mayor densidad poblacional en el aglomerado.

La expansión del aglomerado, por otra parte, a localidades contiguas implicó la integración de áreas de residencia de sectores medios y medios-altos. En el caso de Vaqueros, se observa un notable cambio en el ICVI, entre 2001-2010, que se vincula con su progresiva ocupación por sectores medios en virtud de su característica de comunidad periurbana con marcados rasgos rurales y de precios relativamente accesibles. Las áreas de Cerrillos, 
asimismo, guardan características similares en relación con las condiciones de vida de su población, pero — como se mencionó— se trata de un barrio más antiguo.

El análisis de las características sociodemográficas de la población que vive en áreas de pobreza pone de manifiesto ciertas condiciones de vulnerabilidad que inciden sobre la disponibilidad, distribución y uso de los recursos. Las altas tasas de natalidad y fecundidad y la presencia de hogares numerosos, con mayor presencia de niños, ponen en tensión la necesidad de mayores recursos (ingresos, vivienda, alimentos, trabajo, etc.) y la menor cantidad de miembros que pueden aportar en su satisfacción. El bajo nivel educativo de los/las jefes/ as de hogar condiciona, por su parte, las posibilidades de conseguir una inserción laboral calificada, y, por lo tanto, mejor remunerada, e incide en el clima educativo del hogar.

Los distintos aspectos analizados permiten, en definitiva, demostrar que la pobreza urbana se estructura a partir de las características que asume, en los distintos momentos históricos, el funcionamiento del mercado de trabajo y del mercado inmobiliario. La tendencia claramente es a la precariedad y expulsión de mayores sectores de población y, por lo tanto, al agravamiento de las condiciones de privación. El Estado, por su parte, en sus distintas actuaciones ha favorecido o acompañado las dinámicas de dichos mercados, aun cuando propició políticas habitacionales, como lo demuestran las intervenciones citadas en el trabajo.

Es en este cuadro en que se destacan las estrategias que desarrollan las poblaciones afectadas para acceder a la ciudad y hacer frente a las privaciones. El acceso a loteos, la ocupación en villas o asentamientos, pero antes el alquiler en conventillos e inquilinatos o los primeros ranchos en las afueras de la ciudad son las salidas que encontraron a este entramado. Sin embargo, la persistencia de estos modos de vivir la ciudad y la expansión e intensificación de la pobreza y la acentuación de las desigualdades abren numerosos interrogantes sobre las posibilidades de revertir estos procesos.

\section{Referencias bibliográficas}

ACRECHE, Noemí; ALBEZA, María Virginia y CARO, Fabiana (2011). Biodemografía en la Ciudad de Salta: su población a mediados del Siglo XIX. En Andes, vol. 22, N. ${ }^{\circ}$ 2, pp. 1-14. Recuperado de http://portalderevistas.unsa.edu.ar/ojs/index.php/ Andes/article/view/129/118 
AGUILAR, María Ángela (1987). El estado, la financiación del hábitat y la renta del suelo. El caso Salta. En: Revista Medio Ambiente y Urbanización, N. ${ }^{\circ}$ 21, año 6, pp. 39-48. Buenos Aires: IIED/AL.

AGUILAR, María Ángela; SBROCCO, María Eugenia; VÁZQUEZ, Estela; ÁLVAREZ LEGUIZAMÓN, Sonia; SACCHI... CID, Juan Carlos (2002). Miradas y visiones sobre la Pobreza. Un estudio de la pobreza en la Provincia de Salta en la década del noventa. 2do. Premio del Concurso Nacional, Programa la "Deuda Social". Salta: Universidad Católica Argentina.

AGUILAR, María Ángela; ÁLVAREZ LEGUIZAMón, Sonia y SBROcco, María Eugenia (2000). Sectores populares urbanos y calidad de vida. El caso de los loteos económicos en Salta. Procesos de urbanización en Argentina: la mirada antropológica. San Salvador de Jujuy Editores: Rabey, M. y Jerez, O. UNJu.

AGUILAR, María Ángela y CosTILLA, Miguel (2009). Miradas sobre fronteras en un espacio local. En: Frontera norte, Vol. 21, N. ${ }^{\circ}$ 41, pp. 53-75. Recuperado de http://www.scielo.org.mx/scielo.php?script=sci_arttext\&pid=S0187-73722 009000100003\&lng=es\&tlng=es

AGUILAR, María Ángela y SBROCCO, María Eugenia (1997). Salta frente al pos-FONAVI. Una historia circular. En: B. Cuenya y A. Falú (comp.), Reestructuración del Estado y Política de vivienda en la Argentina. Buenos Aires: Colección CEACBC, UBA, N. ${ }^{\circ} 15$.

AGUILAR, María Ángela y SBROCCO, María Eugenia (2009). Transformaciones e improntas urbanas en un espacio local. El caso de Vaqueros, Salta. En Cuaderno Urbano. Espacio, Cultura, Sociedad, Vol. 8, N. ${ }^{\circ}$ 8. Corrientes: Universidad Nacional del Nordeste, pp. 159- 180. ISNN 1666-6186.

ALTIMIR, Oscar (1979). La dimensión de la pobreza en América Latina. En: Serie Cuadernos de la CEPAL, N. ${ }^{\circ}$ 27. CEPAL: Santiago de Chile.

ÁLVAREZ LEGUIZAMÓN, Sonia (2004). La pobreza: configuraciones sociales, relaciones de tutela y dispositivos de intervención. (Salta primera mitad del siglo XX). En: S. A.C. Rodríguez y S. Álvarez Leguizamón, Abordajes y Perspectivas. Secretaría de Cultura, Ministerio de Educación de la Provincia de Salta, pp. 87-213.

ÁLVAREZ LEGUIZAMÓN, Sonia; GERUZA QUEIROZ, Countinho y ÁLVAREZ, Marcela (2010). El higienismo y la construcción del imaginario urbano en Salta a principios del siglo XX: palas y vacunas, hospitales y gacetas. En S. Álvarez Leguizamón, Poder, salteñidad. Saberes, políticas y representaciones sociales. CEPIHA: Salta. 
ARÉVALO, Carla y PAZ, Jorge (2015). Pobreza en la Argentina. Privaciones múltiples y asimetrías regionales”. En: Documentos de Trabajo, 15. Salta: Instituto de Estudios Laborales y del Desarrollo Económico (IELDE).

ARRIAGADA, Camilo (2000). Pobreza en América Latina: Nuevos escenarios y desafíos de políticas para el hábitat urbano. En: Serie Medio Ambiente y Desarrollo, 27. Recuperado de https://repositorio.cepal.org/bitstream/handle/11362/5711/ S00100849_es.pdf?sequence=1\&isAllowed=y

BAYÓN, María Cristina (2012). El "lugar" de los pobres: espacio, representaciones sociales y estigmas en la ciudad de México. En: Revista Mexicana de Sociología, vol. 74, núm. 1, enero-marzo, pp. 133-166. Recuperado de http://www.scielo. org.mx/scielo.php?script=sci_arttext\&pid=S0188-25032012000100005\&lng $=$ es\&tlng=es

BOLETÍN MUNICIPAL. Noviembre de 1941. Salta.

BOLSI, Alfredo y MIGNONE, Aníbal (2009). La pobreza en los aglomerados urbanos del Norte Grande Argentino. En: Bolsi A. y Paolasso P. (comp.), Geografía de la pobreza en el Norte Grande Argentino. Tucumán: UNT-CONICET.

CANCINOS, Hugo (2002). Sobre el imaginario urbano colonial "La muy noble y leal ciudad de Salta”. En: Kairos, Revista de Temas Sociales, año 6, N. ${ }^{\circ} 11$. Recuperado de https://www.revistakairos.org/sobre-el-imaginario-urbano-colonial-la-muynoble-y-leal-ciudad-de-salta/

CORBACHO, Myriam y ADET, Raquel (2002). La historia contada por sus protagonistas. Salta primeras décadas del siglo XX. Maktur: Salta.

DI FABIO ROCCA, Francisco; ALBEZA, María Virginia; BÁRBARA POSTILLONE, María; ACRECHE, Noemí; LAFAGE, Lucía; PAROLÍN, María Laura... AVENA, Sergio (2016). Historia poblacional y análisis antropogenético de la ciudad de Salta. En: Andes. Antropología e Historia, 27, CEPIHA, Salta.

CRAVINO, María Cristina (2008). Los mil barrios (in)formales. Aportes para la construcción de un observatorio del hábitat popular del Área Metropolitana de Buenos Aires. Universidad Nacional de General Sarmiento, Buenos Aires.

GÓMEZ, Roque Manuel (2017) La ciudad de Salta: Urbanismo, arquitectura y sociedad. EUCASAL, Universidad Nacional de Salta.

JANOSCHKA, Michael (2002). Urbanizaciones privadas en buenos Aires: ¿hacia un nuevo modelo de ciudad latinoamericana? En: L. P. Cabrales Barajas, Latinoamérica: países abiertos, ciudades cerradas. Universidad de Guadalajara, México. 
JUSTINIANO, María Fernanda (2008). Poder y riqueza en Salta a fines del siglo XIX: ¿Cuánto de vacas y cuánto de azúcar? En: Revista Escuela de Historia, N. ${ }^{\circ}$ 7, vol. 1. Recuperado de http://www.unsa.edu.ar/histocat/revista/revista0705.htm

MAC DONALD, Joan (2004). "Pobreza y precariedad del hábitat en ciudades de América Latina y el Caribe”. En: Serie Manuales, 38. División de Desarrollo Sostenible y Asentamientos Humanos, Santiago de Chile: CEPAL.

MATA, Sara (1996). El crédito mercantil. Salta a fines del siglo XVIII. En: Anuario de Estudios Americanos, vol. 53, N. ${ }^{\circ}$ 2. Recuperado de http://estudiosamericanos.revistas.csic.es/index.php/estudiosamericanos/article/view/419/425

MORENO, José Luis (2009). Éramos tan pobres... De la caridad a la Fundación Eva Perón. Editorial Sudamericana, Buenos Aires.

NATERA RIVAS, Juan José (1996). Inquilinatos en la ciudad de San Miguel de Tucumán en la primera mitad del siglo XX. En: Población y Sociedad, N. ${ }^{\circ}$ 4, pp. 178-200. Tucumán: Grupo Editor Yocavil.

OIT (2003). Directrices sobre una definición estadística de empleo informal. Documento de la Décimo Séptima Conferencia Internacional de Estadísticos del Trabajo.

ORTIZ DE D’ARTERIO, Patricia y PAOLASSO, Pablo (2005). Una aproximación al estudio del crecimiento de la población del NOA (1980- 2001). Actas de las VII Jornadas Argentinas de Estudios de Población. 475-496. Buenos Aires: AEPA-INDEC.

PAOLASSO, Pablo; LONGUI, Fernando y VELÁZQUEZ, Guillermo (comp.) (2019). Desigualdades y fragmentación territorial en la argentina durante la primera década del siglo XXI. Buenos Aires: Editorial Imago Mundi.

SBROCCO, María Eugenia (1999). Los barrios en la ciudad. En: El Tribuno. Suplemento especial. Aniversario 50 años (1949-1999). Diario El Tribuno, Salta, pp. 185 - 188.

VAPÑARSKY, César (1994). Crecimiento urbano diferencial y migraciones en la Argentina: cambios y tendencias desde 1970. En: Estudios migratorios latinoamericanos, año 9, N. 27 (agosto), pp. 225-260. Buenos Aires: CEMLA.

WINCHESTER, Lucy (2008). La dimensión económica de la pobreza y precariedad urbana en las ciudades latinoamericanas. Implicaciones para las políticas del hábitat. En: Eure, vol. XXXIV, N. ${ }^{\circ}$ 103, pp. 27-47. Recuperado de https://dx.doi. org/10.4067/S0250-71612008000300002

ZICCARDI, Alicia (comp.) (2008). Procesos de urbanización de la pobreza y nuevas formas de exclusión social. Los retos de las políticas sociales de las ciudades latinoamericanas del siglo XXI. Bogotá: Siglo del Hombre Editores, Clacso Crop. 